\title{
VALOR PREDICTIVO DEL GRADO DE DILATACIÓN URETERAL EN EL ÉXITO DE LA CORRECCIÓN ENDOSCÓPICA DEL REFLUJO VÉSICOURETERAL EN PACIENTES PEDIÁTRICOS
}

\author{
Iván Somoza Argibay, Elena Molina Vázquez y Diego Vela Nieto.
}

Servicio de Cirugía Pediátrica. Hospital Materno Infantil Teresa Herrera. Complejo Hospitalario Juan Canalejo.

La Coruña. España.

Resumen.- OBJETIVO: El 30-50\% de lo pacientes pediátricos diagnosticados de infección del tracto urinario inferior (ITU) presenta Reflujo Vésicoureteral (RVU). El tratamiento endoscópico del RVU se ha convertido en una de sus principales armas terapéuticas y su éxito se ha relacionado tradicionalmente con el grado de RVU. Sin embargo en cada uno de los grados de RVU se pueden observar distintos grados de dilatación ureteral. En este estudio pretendemos estudiar la influencia del grado de dilatación ureteral en la curación endoscópica del RVU.

MÉTODOS: De un total de 632 unidades renales refluyentes (URR) tratadas endoscópicamente; realizamos una revisión de los primeros 4 años (1996-1999). Tras descartar los pacientes con RVU secundario (vejiga neurógena, duplicidad) entraron en el estudio 70 pacientes (un total de 105 URR). Mediante un estudio de doble ciego se valoraron las cistografías miccionales (CUMS), se graduó la dilatación ureteral de todos los pacientes en tres grados: leve-normal, moderada y severa. Realizamos diversas comparaciones entre los porcentajes de curación tras la $7^{\underline{a}}$ inyección endoscópica en los tres grados de dilatación ureteral.

RESULTADOS: Tras la valoración de las CUMS mediante el estudio doble ciego; de las 105 URR estudiadas, 3 presentaban RVU de grado I, 12 de grado II, 64 de grado III y 26 de grado IV. Tras graduar la dilatación ureteral había 45 con grado leve-normal, 44 moderada y 16 severa. Al comparar los porcentajes de curación de los RVU de grado III con los de grado $N$, sin considerar el grado de dilatación ureteral, no se encontró significación estadística. Sin embargo sí encontramos diferencias estadísticamente significativas entre los porcentajes de curación de cada uno de los grados de dilatación ureteral: al considerar todas las URR tratadas, al considerar únicamente los RVU de grado III y IV y al considerar sólo los reflujos de grado III.

CONCLUSIONES: La Clasificación Internacional del RVU como factor pronóstico del éxito del tratamiento endoscópico pasa por alto diferencias entre reflujos del mismo grado. Los resultados observados demuestran que el grado de dilatación ureteral debe ser valorado conjuntamente con el grado de reflujo a la hora de pronosticar el éxito del tratamiento endoscópico del RVU.

Palabras clave: Reflujo vesicoureteral. Tratamiento endoscópico. Dilatación ureteral.

Summary.- OBJECTIVES: $30-50 \%$ of pediatric patients with the diagnosis of lower urinary tract infection (UTI) have vesicoureteral reflux (VUR). The endoscopic treatment of VUR has become one of the main therapeutic tools and its success has been traditionally related to the grade of VUR. Nevertheless, 
in each of the VUR grades different grades of ureteral dilation may be observed. The aim of the study is to evaluate the influence of ureteral dilation rate in the cure rate after endoscopic treatment.

METHODS: From a total of 632 renal units with reflux treated endoscopically, we review the first four years (1996-1999). After discarding patients with secondary VUR Ineurogenic bladder, ureteral duplication) 70 patients enter the study (a total of 105 renal units with reflux). Voiding cystourethrograms (VCUG) were evaluated by means of a double blind study, grading ureteral dilation of all patients in three grades: mildnormal, moderate and severe. We performed comparisons between the cure rates of first endoscopic injection in the three grades of ureteral dilation.

RESULTS: After the evaluation of VCUG in the double blind study, from 105 renal units with reflux in the study, three had grade I VUR, 12 grade II, 64 grade III, and 26 grade IV. After grading ureteral dilation there were 45 with mild-normal grade, 44 moderate and 16 severe. The comparison of cure rates for grade III and IV VUR without considering the grade of ureteral dilation did not show statistical significance. Nevertheless, we found statistically significant differences between the cure rates of each grade of ureteral dilation: when considering all renal units with reflux treated, considering all grade III and IV VUR, and considering only grade III reflux.

CONCLUSIONS: The international classification of VUR as a prognostic factor for the success of endoscopic treatment does not considered differences between same degree refluxes. The results observed demonstrate that grade of ureteral dilation must be evaluated in conjunction with grade of reflux at the time of making prognosis about the success of endoscopic treatment of VUR.

Keywords: Vesicoureteral reflux. Endoscopic treatment. Ureteral dilation.

\section{INTRODUCCIÓN}

El reflujo vesicoureteral (RVU) afecta al 30-50\% de los pacientes pediátricos con ITU y es la patología urológica más frecuente en la edad pediátrica; con una prevalencia del $1 \%$ en niños sanos. La asociación entre reflujo, infección y daño renal está bien establecida, existiendo lesiones renales al diagnóstico entre el $30-70 \%$ de los casos (1-3). La nefropatía por reflujo es la causa de insuficiencia renal terminal en el $3-25 \%$ de los niños (4).

El manejo del RVU en niños continúa siendo controvertido. El objetivo de su tratamiento es prevenir las ITU asociadas al reflujo y las cicatrices renales mediante manejo médico o quirúrgico. El tratamiento médico con pro- filaxis antibiótica resuelve el RVU con el tiempo; siendo el porcentaje de curación en reflujos grados III y IV tan bajo como $16 \%$ a los 5 años (4).

La introducción de una tercera alternativa (tratamiento endoscópico) por Matouscheck en 1981 y posteriormente popularizada por Puri y O'Donnell como "STING" en 1984, se ha establecido y popularizado especialmente en Europa y se ha aceptado como una buena alternativa a la cirugía abierta (4-12).

El RVU es una de las causas principales de nefropatía en niños, pero existen distintos grados de severidad que se correlacionan con el pronóstico (13). La graduación del reflujo nos permite diferenciar los reflujos con alto riesgo de daño renal de aquellos con bajo riesgo (14).

Con la Clasificación Internacional del Reflujo (IRSC) publicada por Lebowitz se ha conseguido una uniformidad en la graduación de la severidad del RVU que es esencial para la comparación de los diferentes métodos terapéuticos. La estandarización de el método de realización de la cistografía consigue evitar diferencias en la técnica que pudieran influir en la determinación del grado de reflujo (13). Según IRSC; los reflujos de grado IV se diferencian de los de grado III básicamente por el abombamiento de los cálices, sin embargo hemos observado grandes diferencias de dilatación ureteral dentro del mismo grado de reflujo (Figuras 1 y 2 ).

En 1995 comenzamos a utilizar el tratamiento endoscópico en nuestro hospital en:

- RVU de grado II tras 1 año de tratamiento médico sin respuesta.

- RVU de grado III en todos los casos.

- RVU de grado IV sin nefropatía de reflujo.

El éxito global tras la primera inyección se sitúa alrededor del $76 \%$ y se considera que el éxito del manejo endoscópico está influenciado fundamentalmente por el grado de reflujo; así existen diversos estudios que demuestran diferentes porcentajes de curación para cada grado de reflujo $(8,12,15)$.

Existen pocos estudios sobre otros factores que potencialmente pueden influir en el éxito del tratamiento (16-17). intentamos conocer los límites del tratamiento endoscópico con el propósito de buscar las mejores opciones terapéuticas para cada paciente con RVU. Así en este trabajo pretendemos estudiar la relación entre la dilatación ureteral y la curación endoscópica del RVU; y valorar si el grado de dilatación ureteral puede ser considerado independientemente del grado de reflujo como un factor determinante del pronóstico endoscópico.

\section{MATERIAL Y MÉTODOS}

Desde 1995 hemos tratado endoscópicamente en el Hospital "Juan Canalejo" de La Coruña un total de 632 unidades renales refluyentes (URR). Realizamos una revisión de los primeros 4 años (1996-1999) recogiendo 81 pacientes. Tras descartar a los pacientes con vejiga neu- 

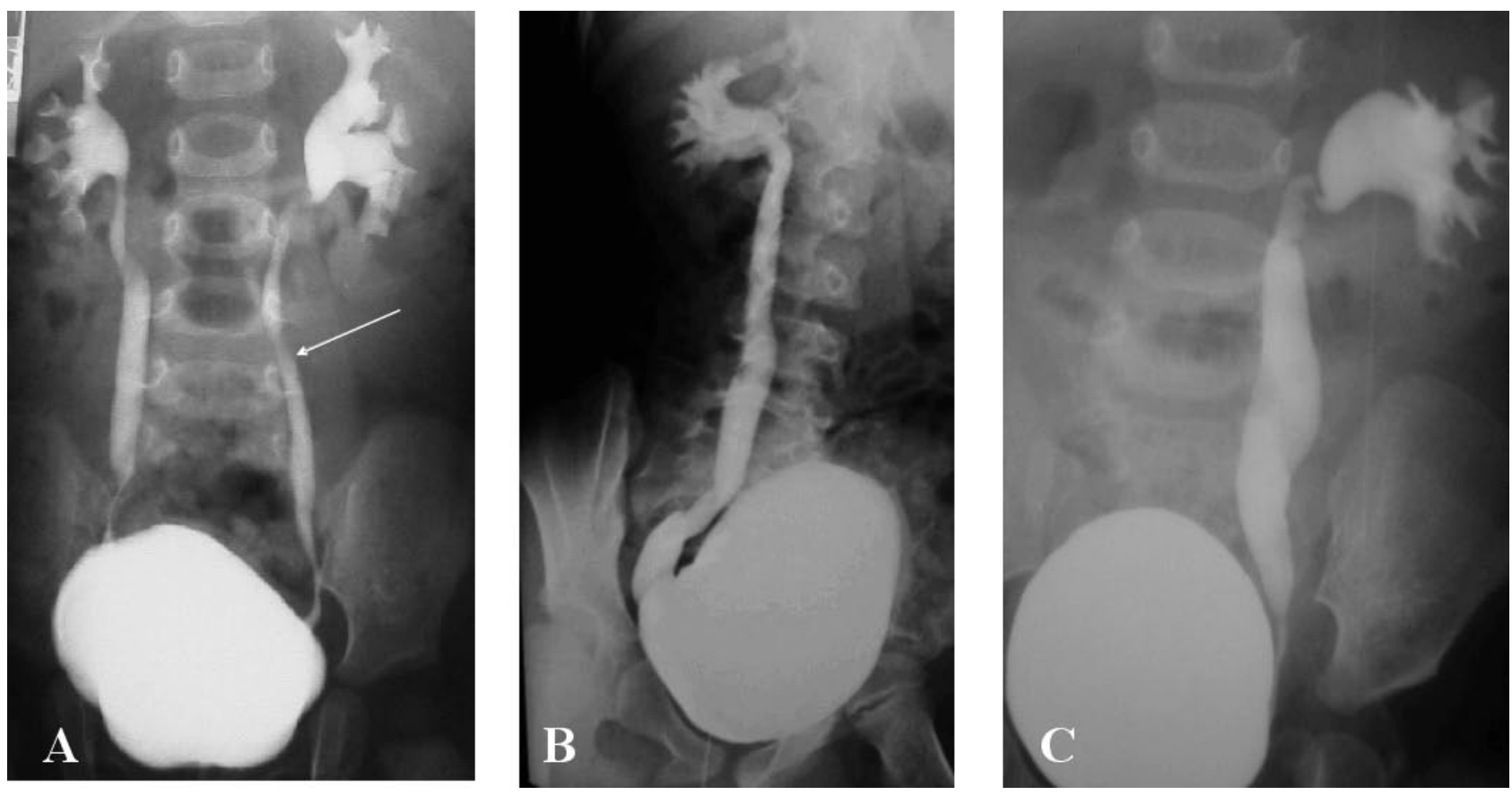

FIGURA 1. RVU de grado III con distinto grado de dilatación ureteral: leve-normal (A), moderado (B), severo (C).

rógena (5) y con duplicidad ureteral (6); valoramos a 70 pacientes con un total de 105 URR que fueron sometidas al estudio independientemente.

Realizamos un estudio de doble ciego con dos especialistas en urología infantil que valoraron las CUMS previas a la primera intervención endoscópica de todos los pacientes sin conocer el resultado del tratamiento endoscópico:

- En primer lugar graduaron las cistografías según la Clasificación Internacional del RVU

- En segundo lugar graduaron la dilatación ureteral en tres
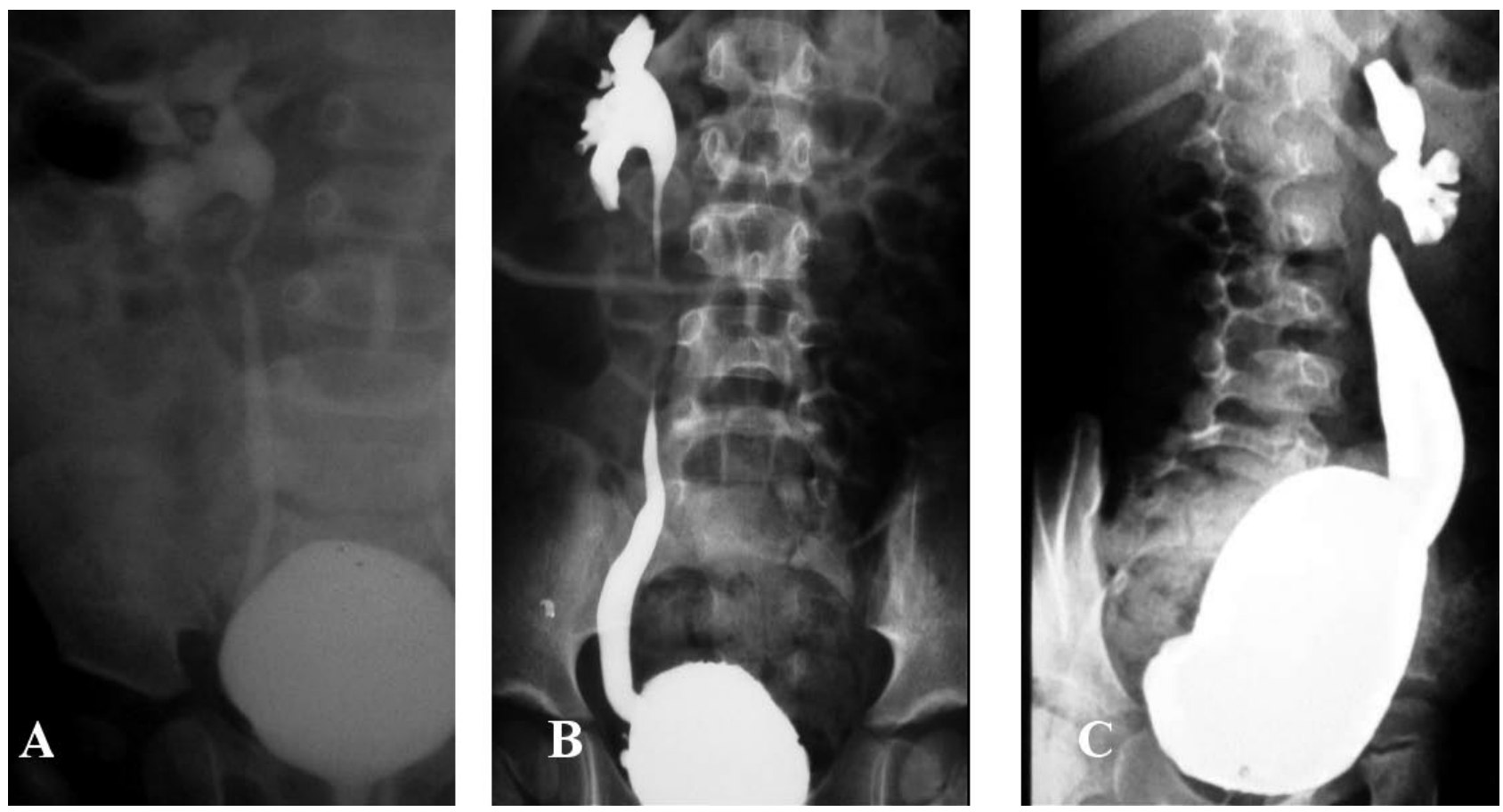

FIGURA 2. RVU de grado IV con distinto grado de dilatación ureteral: leve-normal (A), moderado (B), severo (C). 
grados, tomando como referencia la dilatación máxima ureteral en la CUMS (14):

- Normal-leve

- Moderada

- Severa

En este estudio consideramos curación a la desaparición del RVU en la cistografía de control realizada a los 3 meses de la $1^{\underline{a}}$ inyección endoscópica. Una vez finalizado el estudio de doble ciego de análisis de las cistografías llevamos a cabo el estudio estadístico realizando las siguientes comparaciones entre los porcentajes de curación de distintos grupos de pacientes:

- Comparamos la curación entre los RVU de grado III con los de grado IV.

- Comparamos la curación entre los distintos grados de dilatación ureteral (normal-leve, moderada y grave).

- Comparamos la curación entre los distintos grados de dilatación ureteral considerando únicamente los RVU de grado III y IV.

- Comparamos la curación entre los distintos grados de dilatación ureteral dentro de los reflujos de grado III.

En el estudio estadístico los datos fueron analizados mediante la prueba estadística $\chi^{2}$ para comparación de proporciones, valorando la significación estadística para una $p<0.05$.

\section{RESULTADOS}

El $50 \%$ de los 70 pacientes sometidos al estudio presentaban RVU bilateral, haciendo un total de 105 URR.

TABLA I. DISTRIBUCIÓN DE LOS PACIENTES DEL ESTUDIO SEGÚN EL GRADO DE DILATACIÓN URETERAL Y EL GRADO DE REFLUJO CON LOS PORCENTAJES DE CURACIÓN DE CADA GRUPO.

\begin{tabular}{|c|c|c|c|c|c|}
\hline $\begin{array}{c}\text { R.V.U } \\
\text { DILATACIÓN }\end{array}$ & I & II & III & IV & V \\
\hline LEVE & $\begin{array}{c}3 \\
(100 \%)\end{array}$ & $\begin{array}{c}12 \\
(100 \%)\end{array}$ & $\begin{array}{c}23 \\
(91.3 \%)\end{array}$ & $\begin{array}{c}7 \\
(85.7 \%)\end{array}$ & \\
\hline MODERADA & & & $\begin{array}{c}31 \\
(64.5 \%)\end{array}$ & $\begin{array}{c}13 \\
(46.1 \%)\end{array}$ & \\
\hline SEVERA & & & $\begin{array}{c}10 \\
(40 \%)\end{array}$ & $\begin{array}{c}6 \\
(33.3 \%)\end{array}$ & \\
\hline
\end{tabular}

El $53 \%$ eran del lado izquierdo y el $47 \%$ del lado derecho.

La media de edad en el momento del tratamiento fue de 3.9 años (Rango = 5 meses -13 años). El 79\% de los pacientes tratados eran niñas. los siguientes:

Los resultados tras el estudio a doble ciego fueron

- Al valorar el grado de reflujo según la Clasificación Internacional: 3 RVU de grado I, 12 de grado II, 64 de grado III y 26 de grado IV. La concordancia entre ambos observadores fue del $85 \%$; el 15\% restante se reevaluó conjuntamente.

- Al evaluar el grado de dilatación ureteral se encontró: 45 pacientes con dilatación ureteral normal-leve, 44 con dilatación moderada y 16 con dilatación severa. La concordancia entre ambos observadores fue del $90 \%$; el $10 \%$ restante se reevaluó conjuntamente.

Los porcentajes de curación para cada grado de dilatación ureteral se presentan en la Tabla I. Tras realizar las comparaciones estadísticas encontramos:

- Primero comparamos los porcentajes de curación tras el tratamiento endoscópico de los RVU de grado III (70.3\%) con los de grado IV (53.8 \%) sin tener en cuenta el grado de dilatación ureteral; se observan diferencias importantes; pero a pesar de ello no se alcanzan diferencias estadísticamente significativas ( $p>0.05$ ) Tabla II.

- En segundo lugar comparamos los porcentajes de curación entre los grado de dilatación ureteral leve-normal, moderada y severa:

1. Incluyendo todos los pacientes del estudio encontramos diferencias estadísticamente significativas ( $p<0.05)$ Tabla III.

\section{TABLA II. COMPARACIÓN DE LOS PORCENTAJES DE CU- RACIÓN ENTRE LOS GRUPOS DE RVU III Y IV.}

\begin{tabular}{|c|c|c|}
\hline N=90 & III & IV \\
\hline CURADOS & 45 & 14 \\
\hline NO CURADOS & 19 & 12 \\
\hline \% CURACIÓN & $70.3 \%$ & $53.8 \%$ \\
\hline$>0.05$ & & \\
\hline
\end{tabular}


TABLA III. COMPARACIÓN DE LOS PORCENTAJES DE CURACIÓN ENTRE LOS GRADOS DE DILATACIÓN URETERAL LEVE-NORMAL, MODERADA Y SEVERA CONSIDERANDO TODAS LAS URR ESTUDIADAS.

\begin{tabular}{|c|c|c|c|}
\hline N=105 & LEVE & MODERADO & GRAVE \\
\hline CURADOS & 42 & 26 & 6 \\
\hline NO CURADOS & 3 & 18 & 10 \\
\hline \% CURACIÓN & $93.3 \%$ & $59 \%$ & $37.5 \%$ \\
\hline
\end{tabular}

$P<0.05$

2. Incluyendo únicamente los RVU de grado III y IV nuevamente encontramos diferencias estadísticas significativas $(p<0.05)$ Tabla IV.

3. Considerando únicamente los RVU de grado III también encontramos diferencias estadístcas significativas $(p<0.05)$ Tabla V.

\section{DISCUSIÓN}

La inyección endoscópica subureteral de materiales biocompatibles se ha convertido en una alternativa

TABLA V. COMPARACIÓN DE LOS PORCENTAJES DE CURACIÓN ENTRE LOS GRADOS DE DILATACIÓN URETERAL LEVE-NORMAL, MODERADA Y SEVERA DENTRO DE LOS RVU DE GRADO III.

\begin{tabular}{|c|c|c|c|}
\hline $\mathrm{N}=90$ & LEVE & MODERADO & GRAVE \\
\hline CURADOS & 21 & 20 & 4 \\
\hline NO CURADOS & 2 & 11 & 6 \\
\hline \% CURACIÓN & $91.3 \%$ & $64.5 \%$ & $40 \%$ \\
\hline
\end{tabular}

$P<0.05$
TABLA N. COMPARACIÓN DE LOS PORCENTAJES DE CURACIÓN ENTRE LOS GRADOS DE DILATACIÓN URETERAL LEVE-NORMAL, MODERADA Y SEVERA CONSIDERANDO SÓLO LOS RVU DE GRADO III Y IV.

\begin{tabular}{|c|c|c|c|}
\hline N=90 & LEVE & MODERADO & GRAVE \\
\hline CURADOS & 27 & 26 & 6 \\
\hline NO CURADOS & 3 & 18 & 10 \\
\hline$\%$ CURACIÓN & $90 \%$ & $59 \%$ & $37.5 \%$ \\
\hline$P<0.05$ & & & \\
\hline
\end{tabular}

establecida frente a la profilaxis antibiótica prolongada y el tratamiento quirúrgico en el abordaje del RVU. Debemos ofrecer a cada paciente el tratamiento que mejor convenga según las características de su reflujo. Aunque el porcentaje de éxito con la cirugía endoscópica es ligeramente menor que con la cirugía abierta, los padres tienden a inclinarse por el tratamiento endoscópico debido a su menor morbilidad $(15,16)$.

Con el tratamiento endoscópico se han publicado distintos porcentajes de curación para cada grado de reflujo; en estos estudios se demuestra que las posibilidades de éxito disminuyen progresivamente con el grado $(8,12$, 15). Esto convierte el grado de reflujo en un factor determinante del éxito endoscópico. Otros autores han intentado buscar parámetros propios de la técnica de inyección, el sitio exacto de la inyección, el volumen adecuado del material, el desplazamiento del implante, el material utilizado, la presencia de otras anomalías urológicas asociadas, la presencia de reflujo contralateral..., y los resultados no han sido significativos (12,17-19).

La Clasificación Internacional del RVU publicada por Lebowitz es cualitativa y diferencia los reflujos de grado III y los de grado IV básicamente porque en los primeros se conserva la angulación caliciliar y en los segundos existe un abombamiento caliciliar perdiendo su ángulo agudo, aunque mantiene la impresión papilar en la mayoría de los cálices. Lebowitz hace referencia en su publicación a la presencia de uréteres con dilatación leve, moderada y severa dentro de cada uno de estos grados de reflujo (13).

La observación de grandes diferencias de dilatación ureteral dentro de cada grado de la Clasificación Internacional nos ha llevado a plantearnos en qué medida esta dilatación condiciona el éxito del tratamiento endoscópico. Aunque algunos estudios han realizado normogramas de diámetro ureteral (14); hemos clasificado las dilataciones 
ureterales de una manera cualitativa, al igual la Clasificación Internacional del RVU.

En cuanto a la validez de la clasificación cualitativa que planteamos, es importante el resultado del estudio de doble ciego que hemos realizado en el cual encontramos una ligera mayor concordancia entre observadores cuando valoran los grados de dilatación ureteral $(90 \%)$ que cuando valoran el grado de reflujo $(85 \%)$.

Como hemos observado en nuestro estudio se encuentran grandes diferencias de dilatación ureteral entre los RVU tratados endoscópicamente. Al comparar los porcentajes de curación entre los reflujos de grado III y los de grado IV sin tener en cuenta la dilatación ureteral se observan importantes diferencias pero no son suficientes para encontrar en este estudio significación estadística. Sin embargo al comparar las probabilidades de éxito entre los grados de dilatación ureteral leve-normal, moderada y severa encontramos diferencias estadísticamente significativas cuando consideramos todas las URR estudiadas, también si consideramos únicamente los RVU de grados III y IV, y también dentro de los reflujos de grado III (Figura 1). El número de pacientes de grado IV tratados no es suficiente para estudiarlos independientemente, pero aún así se pueden observar diferencias importantes en la curación de los distintos tipos de dilatación ureteral.

La dilatación del uréter medida de forma independiente al grado de reflujo es un factor pronóstico clave en la expectativa de curación del reflujo tratado endoscópicamente. Puede ser cuestionable nuestro intento de intentar diferenciar ambas clasificaciones, pero la diferente valoración obtenida en nuestro trabajo sobre los mismos pacientes evaluados por el mismo especialista sin considerar la pelvis ni los cálices renales ratifica nuestra hipótesis.

Los resultados obtenidos indican que el grado de dilatación ureteral condiciona el pronóstico del tratamiento endoscópico del reflujo. El advenimiento de la cirugía endoscópica y su difusión posterior a la elaboración de la Clasificación Internacional hace que esta pase por alto diferencias significativas entre reflujos del mismo grado. Por lo tanto consideramos que el grado de dilatación ureteral debe ser considerado conjuntamente con el grado de RVU a la hora de pronosticar su curación endoscópica.

\section{BIBLIOGRAFÍA y LECTURAS RECOMENDADAS ("lectura de interés $y^{* *}$ lectura fundamental)}

*1. WEISS, R.; TAMMINEN, M.; KOSKIMIES, O. y cols.: "Characteristics at entry of children with severe primary vesicoureteral reflux recruited for a multicenter, international therapeutic trial comparing medical and surgical management". J. Urol., 148: 1644, 1992.

2. BILCKMAN, J.G.; TAYLOR, G.A.; LEBOWITZ, R.L.: "Voiding cystourethrography as the initial radio- logic study in the child with urinary tract infection". Radiology, 156: 659, 1985.

3. VELA, D.; MONTERO, M.; MÉNDEZ, R. y cols.: "Contraindicaciones relativas para el tratamiento endoscópico de reflujo vésicoureteral". Cir. Pediatr., 13: 141, 2000.

**4. PURI, P.: "Endoscopic correction of vesicoureteral reflux". Curr. Opin. Urol., 10: 593, 2000.

*5. MATOUSCHECK. E.: "Sobre un nuevo concepto para el tratamiento del reflujo vesicoureteral. Aplicación endoscópica de Teflón”. Arch. Esp. Urol., 34: 385, 1981.

6. GREENFIELD, S.O.; GRISWOLD, J.J.; WAN, J.: "Ureteral reimplantation in infants". J. Urol., 150: 1460, 1993.

7. SCHOLTMEIJER, R.J.: "Treatment of vesicoureteral reflux. Results of a prospective study”. Br. J. Urol., 71: 346, 1993.

8. MONTERO, M.; MÉNDEZ, R.; TELLADO, M. y cols.: "Estudio comparativo del tratamiento del reflujo vésico-ureteral en la edad pediátrica: Revisión de una serie de 636 unidades refluyentes". Cir. Pediatr., 12: 144, 1999.

9. KAPLAN, W.E.; DALTON, D.P.; FIRLIT, C.F.: "The endoscopic correction of reflux by polytetrafluoroethylene injection". J. Urol., 138: 953, 1987.

10. FARKAS, A.; MORIEL, E.Z.; LUPA, S.: "Endoscopic correction of vesicoureteral reflux: our experience with 115 ureters". J. Urol., 144: 534, 1990.

11. SAUVAGE, P.; GUEISS, S.; SAUSSINE, C. y cols.: "Analysis and perspectives of endoscopic treatment of vesicoureteral reflux in children with a 20-month follow-up". Eur. Urol., 17: 310, 1990.

12. HERZ, D.; HAFEZ, A.; BAGLI, D. y cols.: "Efficacy of endoscopic subureteral polydimethylsiloxane injection for treatment of vesicoureteral reflux in children: A North American clinical report". J. Urol., 166: 1880, 2001.

**13. LEBOWITZ, R.L.; OLBING, H.; PARKKULAINEN, K.V. y cols.: "International system of radiographic grading of vesicoureteric reflux". Pediatr. Radiol., 15: 105, 1985.

14. HËLLSTRÖM, M.; HJÄLMAS, K.; JACOBBSON, B. y cols.: "Ureteral diameter in low-risk vesicoureteral reflux in infancy and childhood". Acta Radiol. Diagnosis, 27: 77, 1986.

15. OGAN, K.; POHL, H.G.; CARLSON, D. y cols.: "Parental preferences in the management of vesicoureteral reflux". J. Urol., 166: 240, 2001.

**16. ELDER, J.S.: "Guidelines for consideration for surgical repair of vesicoureteral reflux". Curr. Opin. Urol., 10: 579, 2000.

17. CAPOZZA, N.; LAIS, A.; MATARAZZO, E. y cols.: "Influence of voiding dysfunction on the outcome of endoscopic treatment for vesicouretral reflux". J. Urol., 168: 1695, 20002.

18. CAPOZZA, N.; PATRICOLO, M.; LAIS, A. y cols.: "Endoscopic treatment of vesico-ureteral reflux: twelve years' experience". Urol. Int., 67: 228, 2001.

*19. BOSTON, V.: "What factors cause failure and can these factors be predicted?". Read at the International Workshop on VUR, Dublin, Ireland, 6-7 September 2002. 